\title{
Language and calculation within the parietal lobe: a combined cognitive, anatomical and fMRI study
}

\author{
L. Cohen ${ }^{\mathrm{a}, *}, \mathrm{~S}$. Dehaene $^{\mathrm{b}}, \mathrm{F}$. Chochon $^{\mathrm{a}}, \mathrm{S}$. Lehéricy $^{\mathrm{c}}$, L. Naccache $^{\mathrm{b}}$ \\ ${ }^{a}$ Service de Neurologie 1, Clinique Paul Castaigne, Hôpital de la Salpêtrière, 47/83 Bd de l'Hôpital, 75651 Paris Cedex 13, France \\ ${ }^{\mathrm{b}}$ INSERM U334, Service Hospitalier Frédéric Joliot, CEA/DSV, Orsay, France \\ ${ }^{\mathrm{c}}$ Service de Neuroradiologie, Hôpital de la Salpêtrière, Paris, France
}

Received 14 December 1998; received in revised form 29 November 1999; accepted 22 December 1999

\begin{abstract}
We report the case of a patient (ATH) who suffered from aphasia, deep dyslexia, and acalculia, following a lesion in her left perisylvian area. She showed a severe impairment in all tasks involving numbers in a verbal format, such as reading aloud, writing to dictation, or responding verbally to questions of numerical knowledge. In contrast, her ability to manipulate nonverbal representations of numbers, i.e., Arabic numerals and quantities, was comparatively well preserved, as evidenced for instance in number comparison or number bisection tasks. This dissociated impairment of verbal and non-verbal numerical abilities entailed a differential impairment of the four arithmetic operations. ATH performed much better with subtraction and addition, that can be solved on the basis of quantity manipulation, than with multiplication and division problems, that are commonly solved by retrieving stored verbal sequences. The brain lesion affected the classical language areas, but spared a subset of the left inferior parietal lobule that was active during calculation tasks, as demonstrated with functional MRI. Finally, the relative preservation of subtraction versus multiplication may be related to the fact that subtraction activated the intact right parietal lobe, while multiplication activated predominantly left-sided areas. (C) 2000 Elsevier Science Ltd. All rights reserved.
\end{abstract}

Keywords: Aphasia; Acalculia; Gerstmann's syndrome; Dyslexia; Imaging

\section{Introduction}

In 1920, Henschen, in the same publication in which the term acalculia was coined, claimed that "the calculation ability is a highly composite cerebral function that results from the collaboration of various posterior areas of the left hemisphere" [26]. A wealth of recent studies of numerical abilities in animals, infants, healthy and brain-lesioned adults largely supports this modular approach at the cognitive and at the anatomical level, and confirms that parietal areas are crucial to number processing (see review in Refs. [18,20]). A major distinction that cuts across numerical abilities is

\footnotetext{
* Corresponding author. Tel.: +33-1-42-16-18-49/02; fax: +33-144-24-52-47.

E-mail address: laurent.cohen@psl.ap-hop-paris.fr (L. Cohen).
}

whether they are contingent or not upon the language faculty. It has been shown that animals and preverbal infants possess a variety of number processing abilities, such as the capacity to match numerosities within and across perceptual modalities [8,37] and perform elementary arithmetic computations $[5,41]$. However, the range of numerical abilities widens dramatically as soon as children acquire language, which allows them to associate verbal labels to any precisely defined quantity. Children are then able to develop rich procedures for manipulating numbers in symbolic form, in particular through the mastery of the written language, and following formal mathematical training in school. However, the precise functional and anatomical interplay of numbers and language in human adults is still in many respects poorly understood.

It is clear that some aspects of number processing are only by-products of general verbal abilities. Such is 
the case of the abilities to utter, read, write, or repeat number words, which are not expected to differ much from ordinary words as far as such input, output, or transcoding processes are concerned (see discussion in Refs. [11,12]). A more debated issue, which is the focus of the present study, is the exact role played by verbal processes in elementary arithmetic. We do not refer here to the verbal processes involved in understanding the operands of arithmetic problems or in eventually producing a response, but to verbal processes putatively involved in the computation or retrieval of the appropriate result. We suggested previously, as part of the triple-code model of number processing, that number facts that have been learned by rote at school, first and foremost the overlearned multiplication table, are retrieved as automatic verbal associations [18]. In Henschen's terms, "the simplest operations, for instance, addition and multiplication of single digits, are generally solved in a completely automatic fashion. We hear the multiplication table internally, and we can utter the result without reflection" [26]. In contrast, subtraction problems, which are not commonly learned by rote, must be solved through mental manipulations of the quantities represented by the operands, or 'semantic elaboration'. The status of addition and division is in principle more ambiguous. While many simple addition problems are memorized in a verbal form like multiplication problems, they can also be solved rapidly using counting and other quantity-driven backup strategies such as referring to 10 (e.g. $6+5=6+4+1=10+1=11$ ).

This conception generates explicit predictions concerning: (1) the possible patterns of dissociation between operations in brain-damaged patients; and (2) the relationships between, on the one hand, arithmetic abilities and, on the other hand, general verbal and quantity manipulation abilities. In contrast, most other neuropsychological models of mental arithmetic are actually neutral regarding these two points. Thus the main alternative model, proposed by McCloskey and his colleagues $[14,30]$, postulates that all arithmetic problems are solved on the basis of a single abstract representation of number meaning, and that the four arithmetic operations are supported by distinct and potentially dissociable processes [14]. This hypothesis was initially proposed because there seemed to be no clear pattern in the observed dissociations between preserved and impaired operations in brain-lesioned patients.

In fact, however, the functional analysis proposed by the triple-code model has received support from a number of reports of brain-damaged patients with dissociations between operations. In these cases, arithmetic impairments often affect multiplication more severely than subtraction $[14,19,27,33]$, or subtraction more severely than multiplication [19,23], while the performance of all these patients with addition was intermediate between their performance with multiplication and subtraction. The triple-code model accounts naturally for this pattern, and predicts that it should never be possible to find a patient with impaired multiplication and subtraction, yet with relatively preserved addition; nor should it be possible to have a selective impairment of addition relative to multiplication and subtraction. Also regarding the relationships between calculation impairments and deficits outside of the arithmetic domain, other models remain generally silent, while there is some empirical support to the predictions derived from the triple-code model. For instance, we studied two patients who presented the following double dissociation pattern [19]. On the one hand, patient BOO showed a general deficit of verbal automatisms, entailing a severe impairment of multiplication fact retrieval, while subtraction problem solving was relatively spared. On the other hand, patient MAR showed a general deficit of quantity manipulation, entailing a severe impairment of subtraction, while the retrieval of memorized multiplication facts was better preserved. In the present case, the fact that patient ATH's performance was impaired in a variety of verbal tasks (including manipulation of number words) and preserved in quantity manipulation tasks, led us to expect a specific dissociation between multiplication and subtraction. Note that the contrast between operations in terms of their underlying mechanisms (retrieval vs. algorithm) is not an absolute one. Some familiar subtraction facts may in principle be learned by rote and, conversely, multiplication facts may be solved or checked through algorithmic manipulations. Still, the crucial claim derived from the triple-code model is that, in normal subjects, subtraction and multiplication rely differentially on these two types of mechanisms, and therefore, can be doubly dissociated following brain lesions that differentially affect areas devoted to verbal vs. quantity manipulation processes.

This cognitive debate has a counterpart at the level of brain functional anatomy. Roughly stated, the issue is that of the relationships between the brain areas that subserve verbal abilities and those that subserve quantitative number processing, and of the contribution of these structures to arithmetic problem solving. The core language areas, those whose lesion entails a variety of aphasic deficits, associate left perisylvian regions belonging to the frontal lobe (specially Broca's area), to the supero-lateral temporal lobe (specially Wernicke's area), and to the inferior parietal lobule (specially the supramarginal gyrus) [29]. Additional infero-temporal structures are devoted to the visual processing of written words, and their lesion induces pure alexia [4,15]. Finally, a network of subcortical structures and pathways connected to the cor- 
tical language areas are also necessary to verbal processes $[1,3]$. The extent of the areas that subserve number processing is less precisely defined. First, verbal representations of numbers probably rely on the same structures as words in general, i.e., the classical language system outlined earlier. We have postulated that a special role is devoted to cortico-subcortical loops in retrieving verbal automatic associations such as rote arithmetic facts. Second, functional imaging studies in normal subjects and data from braindamaged patients suggest that bilateral cortical regions centred on the intraparietal sulcus play a central role in number processing [7,16,22,34,35]. Studies of patients with acalculia following lesions in the left intraparietal region, frequently in the context of Gerstmann's syndrome, show that the numerical deficit affects primarily quantity manipulations, while verbal routines such as reading aloud or writing to dictation, may be entirely preserved $[19,20,24,38,39]$.

In summary, the left parietal lobe is likely to be crucial in the two major aspects of number processing, i.e., verbal and non-verbal. Furthermore, the absence of any impairment of language and of number transcoding in Gerstmann's acalculia, following lesions in the intraparietal region, indicates that within the parietal lobe verbal and non-verbal processes involve areas that are at least partially nonoverlapping.

We study here the numerical abilities of a patient who presented with aphasia and deep dyslexia following a lesion affecting the classical language areas, including part of the inferior parietal lobule. Firstly, we show that the patient's non-verbal numerical abilities were largely spared, while most tasks involving numbers in a verbal format were impaired. Secondly, we show that, as predicted, this predominantly verbal deficit entailed a disproportionate deficit of multiplication, as compared with subtraction. Thirdly, through a precise study of the lesion's topography, we try to delineate, within the parietal lobe, the areas subserving verbal and non-verbal number processing. Finally, we try to clarify the mechanisms of the patient's intact and impaired performance using functional MRI.

\section{Case report}

ATH was a 55-year-old right-handed woman with 12 years of education. Two years before the present study, she suffered from a left-hemispheric infarct responsible for right hemiparesis, aphasia, and alexia with agraphia. Hemiparesis receded rapidly, with a residual right-sided sensory impairment predominantly affecting the upper limb. There was no left-right disorientation when the present study was carried out. Language improved partially over the following 2 years. Cerebral MRI showed a left perisylvian lesion that will be described in detail later.

ATH received a French version of the Boston Diagnostic Aphasia Examination, and additional picture naming, word reading, and lexical decision tests (see Table 1). Spontaneous speech, although relatively fluent and very informative, was hampered by word finding difficulties and interrupted syntactic structures. The patient could not recite the alphabet beyond letter $\mathrm{F}$ without making errors. When reciting other automatic series such as the months or the days of the week, she hesitated and made occasional errors. Simple auditory comprehension was largely preserved, while complex sentential material yielded comprehension errors. Word repetition was preserved except for a few phonological errors in the most complex items. Repetition of complex sentences was markedly impaired. Word reading was good, while sentence reading yielded frequent

Table 1

Boston diagnostic aphasia examination

\begin{tabular}{|c|c|}
\hline Subtest & Score \\
\hline \multicolumn{2}{|l|}{ Fluency } \\
\hline Articulation rating & $7 / 7$ \\
\hline Phrase length & $5 / 7$ \\
\hline Verbal agility & $5 / 14$ \\
\hline \multicolumn{2}{|l|}{ Automatic speech } \\
\hline Automatized sequences & $5 / 9$ \\
\hline Reciting & $2 / 2$ \\
\hline \multicolumn{2}{|l|}{ Repetition } \\
\hline Words & $7 / 10$ \\
\hline High-probability sentences & $5 / 8$ \\
\hline Low-probability sentences & $4 / 8$ \\
\hline \multicolumn{2}{|l|}{ Writing } \\
\hline Primer-level dictation & $8 / 15$ \\
\hline Sentences to dictation & $2 / 12$ \\
\hline Spelling to dictation & $2 / 10$ \\
\hline Mechanics & $2 / 3$ \\
\hline Serial writing & $42 / 47$ \\
\hline Written confrontation naming & $7 / 10$ \\
\hline Narrative writing & $1 / 4$ \\
\hline \multicolumn{2}{|l|}{ Auditory comprehension } \\
\hline Body-part identification & $19.5 / 20$ \\
\hline Word discrimination & $69 / 72$ \\
\hline Commands & $15 / 15$ \\
\hline Complex ideational material & $7 / 12$ \\
\hline \multicolumn{2}{|l|}{ Naming } \\
\hline Naming of body-parts & $28 / 30$ \\
\hline Confrontation naming & $95 / 105$ \\
\hline Semantic fluency (animals) & $14 / 23$ \\
\hline Responsive naming & $30 / 30$ \\
\hline \multicolumn{2}{|l|}{ Reading } \\
\hline Word reading & $26 / 30$ \\
\hline Sentence reading & $3 / 10$ \\
\hline \multicolumn{2}{|l|}{ Reading comprehension } \\
\hline Comprehension of oral spelling & $3 / 8$ \\
\hline Word recognition & $8 / 8$ \\
\hline Word-picture matching & $9 / 10$ \\
\hline Sentences and paragraphs & $8 / 10$ \\
\hline Symbol discrimination & $10 / 10$ \\
\hline
\end{tabular}


errors, although the patient generally seemed to grasp the general meaning of sentences. Although writing was slightly clumsy due to the patient's sensory impairment, letter shapes were perfectly normal. However, writing was hampered by pervasive letter substitutions and omissions. Note that the patient made no error on the few items of the BDAE assessing the comprehension and production of fingers names. In an additional test, the patient flawlessly named 80 drawings of simple objects, with occasional word finding difficulties.

The patient's word reading behaviour presented several features typical of a moderate deep dyslexia syndrome [13,36]. Firstly, she made occasional semantic errors (e.g. lettuce $\rightarrow$ "salad"), demonstrating some access to the meaning of words that she was unable to read aloud. Secondly, she made more errors with abstract than with concrete nouns $(5 / 15$ and $0 / 15$ errors, respectively, Fisher exact two-tailed $P=0.042$, with words matched in length and frequency). Thirdly, she made more errors with closed-class than with open-class words $(9 / 28$ vs. $2 / 28$, respectively, Fisher exact two-tailed $P=0.040$, with words matched in length and frequency). Fourthly, she made many errors reading aloud even short and simple nonwords (8/15 errors). Finally, her performance in lexical decision tasks was good as compared with her reading performance. She made 3/112 errors with a list comprising the 28 closed-class and 28 open-class words mentioned before, and 56 nonwords differing from the real words by a single letter. She made 4/90 errors with a list comprising 20 real words, 20 nonword homophones of real words, and 20 non-homophone nonwords.

\section{Study of number processing}

\subsection{Preliminary number processing assessment}

Preliminary screening of ATH's numerical abilities revealed a marked deficit in most usual tasks. She made pervasive errors when reading aloud Arabic and spelled-out numerals and when writing them to dictation. She was also impaired on even simple arithmetic problems. According to classical typologies, she should thus be classified as suffering both from alexic/agraphic and from anarithmetic acalculia [25]. Her digit span was 3.5 items. She was asked to repeat 54 numbers of increasing length. She made $0 / 9$ errors with 1 -word and 2-word numerals, 1/9 error with 3-word numerals, and 2/9 errors with 4-, 5-, and 6-word numerals. Counting orally up to 20 was difficult beyond number 10 , and the patient omitted numbers 11 and 17 . Counting backwards was extremely difficult, yielding errors even in the range of single digits (from 20: 20,
$19,18,16 \ldots 18 \ldots 20,21 \ldots 20,19,18,17,16 \ldots 17 \ldots$ no!; from 10: 10, 9, 8, 7, 5, 4, 3, 1, 0). In contrast, she could accurately decide which of two Arabic numerals was larger, a general finding in patients with even the largest left-hemispheric lesions. Finally, the patient reported no substantial difficulties in everyday life situations requiring quantity manipulations.

In order to assess ATH's knowledge of encyclopaedic and personal numerical facts, she was proposed a series of oral questions requiring numerical answers (e.g., How many minutes in an hour? How old are you?). She had frequent word finding difficulties, which she managed to circumvent effectively either by displaying the appropriate number of fingers, or by tracing Arabic numerals with her index finger. Her oral responses were erroneous in $9 / 23$ questions. However, she produced the correct response in Arabic form in all nine cases. For instance, when asked how many days there are in January, she immediately wrote 31 , while saying "ten, twenty, thirty, forty... no, before... twenty, thirty...". When asked how many eggs there are in a dozen, she wrote 12 while saying "sixteen". When asked to indicate the zip code of her region, she correctly wrote 34 , but said "forty... three... one, two, three... forty-four maybe...". Moreover, some of her correct oral responses were actually produced after an initial Arabic or gestural response. For instance, when asked how old she was, she said "ffifty and a bit more", then wrote 54, and eventually said "...fiftyfour".

In summary, this preliminary assessment suggested that while all tasks involving numbers in a verbal format (oral or spelled-out number words) were impaired, ATH was still better able to manipulate Arabic numerals and quantitative knowledge. This dissociation will be evaluated in the following sections.

\subsection{Non-verbal number knowledge}

\subsubsection{Comparison of arabic numerals}

The patient was asked to circle the larger of two Arabic numerals of equal length (1-5 digits). She made no error in 26 trials. In a further set of 86 pairs of 1-5 digit numerals, to be described in a subsequent section, she made $2 / 86$ errors. In a computerized comparison task, she was presented with 1762 -digit numerals. She was instructed to decide whether each number was larger or smaller than 55, and to respond using a joystick. She did not make a single error. Reaction times were fast (mean RT $=785 \mathrm{~ms}$ ), and showed a normal distance effect (regression of RT on the logarithm of distance from 55: $r(173)=-0.45$; $P<0.0001)$.

\subsubsection{Proximity judgement}

ATH was asked to circle which of two 1-digit or 2- 
digit Arabic numerals was closer in magnitude to a third one. She made $1 / 30$ error $(10 \rightarrow 5$ instead of 9$)$.

\subsubsection{Number bisection}

The patient was presented with 16 pairs of Arabic digits, with the smaller digit printed on the left and the larger on the right, and was asked to write down the digit that fell in the middle of the interval (e.g., $17 \rightarrow$ $4)$. She made $12.5 \%(2 / 16)$ errors, erroneously producing the difference of the two digits instead of their mean. She was again presented with the same 16 pairs, and was asked to choose the correct response among three proposed digits. She made no error. In a third version of the task, each stimulus pair was presented with the correct result once, and with a proposed false result next time, in random order. ATH made 4/32 errors in deciding whether the proposed result was correct or not.

\subsubsection{Thermometer}

The patient was presented with 22 vertical lines, 7 $\mathrm{cm}$ high, labelled 0 at the bottom and 100 at the top, and with 8 similar lines labelled 1000 at the top. For each line the patient was asked to write down the Arabic number corresponding to a marked point, as if reading up a thermometer. Responses, which were fast and accurate, were highly correlated with the exact value $(r(28)=0.99)$. The mean absolute error was less than $3 \mathrm{~mm}$. Conversely, the patient was presented with 8 Arabic numerals and asked to point to the corresponding location on lines labelled 0 and 100. Again, responses were very accurate $(r(6)=0.99$; mean absolute error $<2 \mathrm{~mm}$ ).

\subsection{Processing of numbers in verbal form}

\subsubsection{Reading arabic numerals aloud}

ATH was asked to read aloud a total of 103 Arabic numerals 1-5 digit long. She made 51.5\% (53/103) errors, including word substitutions or lexical errors (e.g., $98 \rightarrow$ "ninety nine"), syntactic errors (e.g., $235 \rightarrow$

\footnotetext{
${ }^{1}$ Some of the syntactic error were detectable only through the absence of the 'et' word (e.g., $31 \rightarrow$ 'dix, vingt, trente...et puis un...trente-un' [ten, twenty, thirty, and then one... thirty-one] instead of the correct 'trente et un' [thirty and one]. Some errors even appeared as pure liaison errors. For instance, ATH read the number 28 without making the obligatory liaison of the word 'vingt' and 'huit'. In this latter example, after some trial and error, she managed to select the appropriate words, but could not embed them in an adequate syntactic frame.

${ }^{2}$ In 20 out of the 38 single-word pairs, the two number words belonged to the same lexical class (two ones, two teens, or two tens) and the comparison was expected to be easier. In the remaining 18 pairs, the two number words belonged to different lexical class and had potentially deceitful stack relationship (e.g., in the pair 1430,30 is larger although 3 is smaller than 4)
}

"twenty thirty five"), and mixed errors (e.g., $467 \rightarrow$ "forty six nine").

When attempting to retrieve a given number word, the patient often resorted to a counting strategy (e.g., $30 \rightarrow$ "ten, twenty, thirty!"). It should be noted that the availability of this strategy implies that the patient could readily identify numerals that, however, she could not read aloud in a normal fashion. Furthermore, some of the Arabic stimuli had an encyclopaedic content over and beyond their quantitative meaning. ATH understood such numerals accurately even when she was unable to read them aloud. For instance, when presented with 1998, she responded "it is now, but I cannot say it".

Building-up the syntactic frame of multiword numerals was difficult, specially for numerals over 100 , and most syntactic errors were actually failures to produce any well-formed string of number words. ${ }^{1}$

In summary, patient ATH, although able to access the quantitative and the encyclopaedic meaning of Arabic numerals, was severely impaired at translating them into words. This impairment affected both the selection of individual words within the lexicon, and the build-up of a syntactic frame.

\subsubsection{Writing Arabic numerals to dictation}

The patient was asked to write down in Arabic format 86 orally presented numerals $1-5$ digit long. She made $46.5 \%(40 / 86)$ errors, including lexical errors (e.g., soixante neuf [69] $\rightarrow$ 49), syntactic errors mostly with numerals over 100 (e.g., vingt mille [20000] $\rightarrow$ 200), and mixed errors (e.g., mille vingt neuf [1029] $\rightarrow$ 124). ATH thus presented a similar impairment in translating Arabic numerals to verbal numerals and vice versa.

\subsubsection{Reading spelled-out numerals aloud}

The patient was asked to read aloud 76 spelled-out numerals smaller than 100 . She made $48.7 \%$ (37/76) errors. This error rate was similar to her error rate when she was reading Arabic numerals smaller than 100 (46.8\% (37/79) errors). Her behaviour was also qualitatively similar, with numerous lexical errors, a counting behaviour and some difficulties appreciating syntactic structures (e.g., trente six [35] $\rightarrow$ "dix, vingt, trente, et puis ensuite un, deux, trois, quatre, cinq, six" $[10,20,30$ and then $1,2,3,4,5,6]$; vingt six [26] $\rightarrow$ "vingt six" [20-6] without the mandatory liaison).

\subsubsection{Comparison of verbal numerals}

In order to assess number comparison, a list of 86 pairs of numerals was created. The list comprised 38 pairs of single-word numerals and 48 pairs of multiword numerals. ${ }^{2}$ Multiword pairs included 10 syntactically simple pairs, and 38 syntactically complex pairs. In simple pairs, the comparison could be performed 
Table 2

Number of errors when comparing pairs of syntactically simple or complex numerals in verbal and Arabic notations

\begin{tabular}{llll}
\hline & \multicolumn{2}{l}{ Input modality } \\
\cline { 2 - 4 } & Oral & Spelled-out & Arabic \\
\hline Syntactically simple pairs & & & \\
Single-word numerals & $1 / 38$ & $3 / 38$ & $2 / 38$ \\
Multiword numerals & $2 / 10$ & $1 / 10$ & $0 / 10$ \\
$\quad$ Total & $3 / 48$ & $4 / 48$ & $2 / 48$ \\
Syntactically complex pairs & $12 / 42$ & $6 / 38$ & $0 / 38$ \\
Total & $15 / 90$ & $10 / 86$ & $2 / 86$ \\
\hline
\end{tabular}

correctly on the basis of a word-by-word strategy, without considering the complete syntactic structure of the numeral. For instance, it may be deduced that 'sixty eight' is larger than 'fifty three' simply by considering that 'sixty' is larger than 'fifty'. In contrast, comparison of the complex pairs required that the syntactic structure of the stimuli be fully understood. For instance 'vingt quatre' (24) and 'quatre vingt' (80) are composed of exactly the same words, as are 'cent deux' (102) and 'deux cents' (200). We tried to induce errors with pairs such as 'cent huit' (108) vs. 'quatre cents' (400), 'quatorze mille' (14000) vs. 'mille dixneuf' (1019), or using the peculiarities of the French syntax of $70 \mathrm{~s}, 80 \mathrm{~s}$, and $90 \mathrm{~s}$. Care was taken that the number of words was not a systematic clue to the correct response. The list was shuffled randomly and presented in spelled-out verbal notation, in oral form, and in Arabic notation. ${ }^{3}$

On oral input, ATH made 15/90 errors, 12 of which affected syntactically complex trials (Table 2). On spelled-out input, she made 10/86 errors, 6 of which affected syntactically complex trials. For instance, in both notations, she judged 'mille neuf' (1009) to be larger than 'cinq mille' (5000), and 'mille cent dix huit' (1118) larger than 'mille six cent dix' (1610). Faced with the latter problem, she observed 'there is a rule here which I have lost'. In Arabic notation, the patient made only $2 / 86$ errors, a performance better than with either oral or spelled-out numerals $(P=0.0013$ and $P=0.017$, respectively).

In conclusion, the patient's understanding of elementary number words was spared, ${ }^{4}$ whereas errors in

\footnotetext{
${ }^{3}$ During oral presentation, the experimenter pointed to conventional location on the table on the patient's right and left when uttering the first and second numerals in each pair, respectively. Stimuli were repeated whenever necessary. In order to avoid verbal output difficulties, the patient was asked to respond by simply pointing to the conventional location corresponding to the chosen numeral.

${ }^{4}$ Patient ATH's ability to understand elementary spelled-out number words was confirmed by her flawlcss performance when she was asked to select among an array the written word corresponding to a given Arabic numeral (0/26 error).
}

comparing complex multiword numerals, although not pervasive, indicate that the patient was not fully able to exploit syntactic structure in recovering the meaning of verbally presented numerals.

\subsection{Summary}

Despite the prima facie evidence that patient ATH was severely acalculic, we have shown that numerical processing was largely preserved as long as no coding of numbers in a verbal format was required. When she was presented with Arabic numerals, the patient could access and manipulate the associated quantities, as evidenced by her good performance in comparison, bisection, proximity judgement, and pointing to an analogic scale. Vice versa, she could to a large extent translate semantic knowledge into Arabic numerals, as attested by her ability to answer tests of number knowledge and to express values on an analogic scale using Arabic numerals. ATH also had access to the encyclopaedic content of meaningful Arabic numerals such as familiar dates.

In contrast, a substantial impairment was apparent in most tasks involving numbers in a verbal format. It is not in the scope of this study to explore thoroughly the intricacies of the patient's deficit in the verbal sphere [31]. However, a crude picture of her lost and preserved abilities can be sketched as follows. Firstly, ATH's performance in verbal number comparison suggests that she could access the quantities associated with single number words presented either in their written or in their oral form. This conclusion is further supported by the availability of an effective counting strategy for reading aloud spelled-out numerals. Secondly, the access to number words was impaired in all verbal production tasks, such as reading aloud Arabic or spelled-out numerals, or responding verbally to tests of numerical knowledge. Thirdly, an impairment was apparent whenever the use of syntactically structured verbal numerals was required: comparison of syntactically complex pairs of numbers in verbal format, reading aloud Arabic or spelled-out numerals.

\subsection{Mental arithmetic}

We now turn to evaluating the patient's performance in arithmetic tasks. As described in Section 1, the triple-code model suggests that calculation involves both verbal and non-verbal numerical representations, to various degrees depending on the considered operation. Given that the patient's processing of quantities was relatively more preserved than her processing of verbal numerals, we predicted that language-based arithmetic operations should be more impaired than operations that can be solved using quantity manipulations. In particular, familiar multiplication facts, which 
are typically solved by accessing a memorized table of rote verbal associations, should be highly error-prone, while subtraction facts of similar complexity should remain better preserved.

\subsubsection{Preliminary assessment of arithmetic abilities}

As part of the preliminary assessment of the patient's numerical abilities, she was presented with simple arithmetic problems in Arabic notation, and was asked to write down the corresponding results. Stimuli included 20 multiplication problems with single-digit operands, 20 subtraction problems and 20 addition problems with 1- or 2-digit operands. Her performance was good both with subtraction and addition problems $(2 / 20$ and $1 / 20$ errors, respectively), while she was severely impaired with multiplication problems (10/20 errors).

This dissociation between multiplication and subtraction was thus in agreement with our expectations. In the following, we will further evaluate this prediction using problems more carefully matched across the different types of operations.

\subsubsection{Comparison of operation types using matched elementary problems}

ATH was presented auditorily with simple addition, subtraction, and multiplication problems, and was asked to produce the result orally. The set of subtraction problems comprised all 36 possible pairs of operands 1 through 9 with a strictly positive result. The same 36 pairs of operands were used for the multiplication and the addition problems. In multiplication problems, the order of the two operands was reversed in order to present the problems in the more familiar order (smaller operand first). Whenever the patient produced several successive responses on a given trial, which occurred rarely, only the last response was scored. Spontaneous self-corrections were therefore scored as correct responses.

Error rates differed widely across operation types $\left(\chi^{2}(2)=34.4, \quad P<0.0001\right.$; see Table 3). Subtraction yielded only $8.6 \%$ errors, while multiplication yielded as much as $75 \%$ errors $\left(\chi^{2}(1)=32.9\right.$, $P<0.0001)$. Performance with addition problems was worse than with subtraction problems $\left(\chi^{2}(1)=6.8, P=0.009\right)$, but better than with multi- plication problems $\left(\chi^{2}(1)=12.6, \quad P<0.0004\right)$. The patient generally responded with apparent ease, albeit abnormally slowly, to subtraction problems. With multiplication problems, she was quite aware of her difficulties, and often declared that she was just guessing. With addition problems, she often resorted to manual procedures in order to overcome oral output difficulties in formulating the result: she would either trace the result in Arabic code with her index finger, or show the corresponding number of digits. On the whole, her gestural responses were more accurate than her oral responses: if we take into account correct gestural responses, the error rate drops from $33.3 \%$ to $16.7 \%$ (6/36). For instance, when presented with $7+5$, ATH said "a dozen... eleven perhaps", while showing 12 (10 and 2) fingers. When attempting to solve the problem 8 +5 , she said "fourteen, the number that carries good luck!", while tracing with her finger the Arabic numeral 13. A similar behaviour was observed with subtractions. As she was asked to solve the orally presented problem $9-1$, the patient immediately drew with her index finger the correct response 8, and then had to count verbally from "one" to "eight" in order to utter the correct answer. In the context of arithmetic processing, two main indications may be drawn from the patient's gestural behaviour. Firstly, it shows that some of her difficulties with oral arithmetic were obviously related to her impaired verbal output. Secondly, the concomitant production of a fast and correct nonverbal response, and of a wrong, delayed, or even absent verbal response suggests that the patient did not rely on verbal counting for computing the correct solution. In order to compare her performance across different operations while avoiding confounds with word finding difficulties, the same sets of problems were presented to the patient in Arabic notation, and she was asked to write down the results. ATH was also asked to solve 36 division problems derived from the set of multiplication problems. Like in the oral modality, subtraction was almost flawless $(2.8 \%$ errors), while multiplication yielded $47.2 \%$ errors $\left(\chi^{2}\{1\}=19.0, P<0.0001\right)$. It took her as long as $9 \mathrm{~min}$ to solve the 36 multiplication problems. Addition problems were solved as accurately

Table 3

Error rates in matched elementary arithmetic problems

\begin{tabular}{|c|c|c|c|c|}
\hline & Subtraction & Addition & Division & Multiplication \\
\hline Auditory $\rightarrow$ Oral & $3 / 36(8.6 \%)$ & $12 / 36(33.3 \%)$ & & $27 / 36(75.0 \%)$ \\
\hline \multicolumn{5}{|l|}{ Arabic $\rightarrow$ Arabic } \\
\hline Matched operands & $1 / 36(2.8 \%)$ & $1 / 36(2.8 \%)$ & $22 / 36(61.1 \%)$ & $17 / 36(47.2 \%)$ \\
\hline Matched result & $1 / 36(2.8 \%)$ & $5 / 36(13.9 \%)$ & & \\
\hline
\end{tabular}


as subtraction problems (2.8\% errors). Division problems yielded a high rate of errors $(61.1 \%)$, not different from the rate of multiplication errors $\left(\chi^{2}(1)=1.40, P=0.24\right)$.

It could be argued that the difference in performance across operation types was not due to a properly arithmetic cause, but simply to the fact that subtraction results were always single digits, while addition results and even more multiplication results often consisted of multidigit numerals. We therefore presented to the patient sets of subtraction and addition problems whose results were identical to the results of the above multiplication problems. In order to keep the operands as similar as possible to the single digit operands of multiplication problems, subtraction problems were of the type $(10 \times n)-m$ with $m$ in the $0-10$ range. Hence these subtraction problems comprised only two significant digits, as did the corresponding multiplication problems. ATH still made only a single subtraction error, and it took her 5 min to solve the 36 problems. She made $13.9 \%$ addition errors, a performance in the range of the above addition tasks. Thus arithmetic tasks in the Arabic modality confirmed that the dissociation between a severe impairment in multiplication and a relative preservation of subtraction abilities was not simply a by-product of the patient's output difficulties. Still, it is likely that the larger effect of operation type that was observed in the oral modality than in the Arabic modality resulted from the greater verbal complexity of multiplication results as compared with subtraction and addition results.

\footnotetext{
${ }^{5}$ Patient ATH was in many respects similar to the aphasic patient studied by Cohen et al. [11]. The two patients displayed a similar impairment in handling numbers in a verbal format (deep dyslexia for words and numerals), contrasting with preserved non-verbal number processing abilities. The assessment of arithmetic abilities received little emphasis in Cohen et al.'s [11] study. Still the patient was able to decide accurately whether addition problems were correct or false. Other operations were not systematically tested. However, clinical data not included in the original article suggest that all operations were not affected to the same extent. The patient solved flawlessly 8 addition and 8 subtraction problems with 1- or 2-digit opeands, while he made $5 / 8$ error in elementary multiplication problems with 1-digit operands, thus conforming to the same pattern as documented in patient ATH.

${ }^{6}$ A closer analysis of ATH's calculation errors is also suggestive of preserved quantity manipulation abilities: even when she erred, her responses were relatively close to the correct result. In subtraction problems, she was wrong by 1 unit in 3 error trials, and by 2 unit in the remaining 2 error trials. When she was asked to solve multiplication problems, she tried to retrieve the result from her memory. As mentioned before, she was well aware of the impairment of this procedure. She then either refused to propose any response, or accepted to guess. When she was guessing, her responses were sometimes perseverative (e.g., she responded "thirty" erroneously four times in a series of six consecutive trials of oral multiplication). Still, the magnitude of her erroneous multiplication responses was correlated with the size of the correct product $(r(27)=0.64 ; p=0.0002)$.
}

\subsection{Discussion}

Our goal was to determine whether a patient with dissociated quantity and verbal number processing would show a dissociation between operation types, as predicted by the triple-code model. ${ }^{5}$ Multiplication problems stored as automatic verbal associations were expected to be more error-prone than subtraction problems, which are thought to be often solved on the basis of quantity manipulations. This prediction was supported by the data. Patient ATH showed a severe deficit with even the simplest multiplication problems, while she was much better at solving subtraction problems of matched verbal complexity. ${ }^{6}$ Her performance level with addition and division was comparable to her performance level with subtraction and multiplication, respectively.

We cannot exclude that the patient occasionally resorted to a counting strategy for solving addition and subtraction problems, although the clear superiority of her gestural over her verbal responses indicates that verbal counting cannot account for the general sparing of these operations. Note also that the rarity of self-corrections ( 5 out of a total of 108 subtraction problems, possibly including 2 or 3 purely graphic problems) suggests that the preservation of subtraction relative to multiplication did not result from a selective use of compensatory counting-based strategies. Reaction time data, which could have definitely ruled out a counting-based interpretation, were unfortunately not gathered in this study.

At any rate, however, we note that a putative contribution of counting to ATH's performance would not run against our general interpretation that semantic quantity processing was preserved in patient ATH. While counting strategies require that rote verbal sequences (e.g., "one, two, three...") be available, such sequences must be used in the context of appropriate quantity-driven problem-solving procedures. For instance, in order to solve a subtraction problem (e.g., $7-2$ ) through forward counting (e.g., " $2 \ldots 3,4,5,6$, $7 \ldots$ 5!"), one must still understand the meaning of the operation in order to select the appropriate procedure, select the larger operand, etc. [23]. Solving problems through counting thus involves both pure verbal memory and quantity manipulation abilities. This was illustrated most clearly by the case of patient MAR, who could count easily, but was utterly unable to solve even simple subtraction problems, or to bisect numbers, due to a conceptual quantity manipulation deficit [19]. Thus, even if part of ATH's preserved performance in addition and subtraction was due to counting, this would still imply that her semantic understanding of numerical quantities and their relations was partially preserved.

The quantity representation which is presumably 
involved in the patient's residual arithmetical abilities, is thought to encode quantities as distributions of activation on a internal number line, with overlap of the distributions for close numbers [17]. This property can explain that ATH's responses, even when erroneous, were always numerical close to the correct response. A more puzzling aspect of ATH's subtraction performance was its remarkable accuracy, which may seem incompatible with the postulated imprecision of the internal representation of quantities. However, there is converging evidence that the variance of the activation distribution is proportional to the magnitude of the considered number [20]. For small numbers, the distributions that encode two nearby quantities have little overlap, which may allow for a relatively good accuracy in the small arithmetic problems used here. Furthermore, even for larger numbers, higher accuracy can be obtained at the expense of longer response time by resampling from the same variable distribution, as demonstrated in random walk models of number comparison $[6,20]$. Finally, it is possible that the quantity representation, through development, education, and exposure to exact numerical symbols, becomes more accurate in human adults than it is in animals. Indeed, the Weber fraction for numerosity, measured with similar methods, appears consistently much smaller in humans than in animals [40]. Thus, intact quantity processing may suffice to explain ATH's accurate subtraction performance, though we cannot exclude a small contribution of counting-based strategies.

\section{Anatomical and functional mechanisms of the deficit}

We now turn to an anatomical and functional magnetic resonance study of patient ATH. The triple-code model postulates that the brain areas involved in number processing include a left perisylvian region devoted to verbal processing, and a more dorsal bilateral intraparietal region where quantities are represented. Considering that the cognitive impairment of patient ATH affected verbal processes, we expected that language areas would be affected, while the intraparietal region would be spared by the lesion. Furthermore, we expected the left and right intraparietal regions to be activated in patient ATH during number processing, as is found in normal subjects $[7,22,35,36]$. Finally, we explored whether significant activation differences could be found for multiplication versus subtraction, which might explain their behavioral dissociation.

Three tasks were therefore devised: subtraction verification, multiplication verification, and a control task of letter matching. During the calculation tasks, the patient decided whether simple arithmetic operations were true or false. The control task was designed to include similar visual input, response decision and motor processes, but no calculation component. Two considerations prompted us to use problem verification tasks during fMRI scanning instead of the explicit production of arithmetic facts. Firstly, as described before, the patient suffered from substantial word-finding difficulties in the number domain. We judged that the patient would not be able to respond using inner speech at the fixed and relatively rapid rates of trial succession required by fMRI paradigms. Secondly, verification tasks with motor responses allowed us to collect behavioral data during fMRI scanning. Before the actual fMRI acquisition, the patient performed the three tasks out of the scanning machine as a training session.

\subsection{Method}

\subsubsection{Stimuli}

The sets of subtraction, multiplication, and letter matching problems each consisted of 20 true problems mixed with 20 false problems. In multiplication problems, both operands were single digits, and the result was a 2-digit number. The proposed results of false problems were within-table numbers numerically close to the true result (e.g., $4 \times 6=25$ ). In subtraction problems, the first operand was a number in the 11-19 range, the second operand and the result were single digits. The proposed results of false problems were on the average false by 2.65 units. In letter matching problems, the patient was presented with two letters separated with a dot, and followed by an arrow and two joined letters. In correct problems, the letters were the same at the left and right of the arrow (e.g., A. B $\rightarrow$ $\mathrm{AB}$ ), while in false problems, one or two of the letters differed (e.g., A. B $\rightarrow$ AC; A. B $\rightarrow$ EG). Each problem was presented for $2500 \mathrm{~ms}$, and followed by a $2500 \mathrm{~ms}$ blank screen. Due to the sensory deficit affecting her right hand, the patient felt unable to use this hand properly in the scanning machine. Therefore, she simply pressed a left-hand key on correct trials, and did not respond on false trials.

\subsubsection{Image acquisition}

For each task, a series of trials consisted of $12 \mathrm{~s}$ of initial rest, followed by five blocks of 8 trials each ( 40 s) alternating with five blocks of $40 \mathrm{~s}$ rest, for a total of $412 \mathrm{~s}$. The patient performed two subtraction series, two multiplication series, and one letter matching series. In each series, 103 functional volumes sensitive to blood oxygen level dependent contrast were acquired with a T2*-weighted gradient echo, echo planar imaging sequence $\left(\mathrm{TR}=4000 \mathrm{~ms}, \alpha=90^{\circ}\right.$, TE $=$ $60 \mathrm{~ms}$, field of view $=240 \times 240 \mathrm{~mm}$, inplane resolution $=3.75 \times 3.75 \mathrm{~mm}^{2}$ ). Each volume comprised 26 axial slices of $5 \mathrm{~mm}$ thickness covering most of the brain. The first three volumes were discarded to reach 
signal equilibrium. High-resolution images (3D fast gradient-echo inversion-recovery sequence, $\mathrm{TI}=600$ $\mathrm{ms}, \mathrm{TR}=1100 \mathrm{~ms}, \mathrm{TE}=2 \mathrm{~ms}, \alpha=20^{\circ}$, field of view $=240 \times 240 \mathrm{~mm}$, slice thickness $=1.5 \mathrm{~mm}$, inplane resolution $=0.94 \times 0.94 \mathrm{~mm}^{2}$ ) were also acquired for anatomical localization.

\subsubsection{Statistical analysis}

The anatomical images were visualized using a commercial package (VoxTool, General Electric, Milwaukee). The functional images were analyzed with the Statistical Parametric Mapping software (SPM96). To correct for motion, functional scans were realigned using as a reference the last functional image, which was temporally closest to the acquisition of the anatomical image. The anatomical image was transformed stereotactically to Talairach coordinates using the standard template of the Montreal Neurological Institute. The functional scans were then normalized using the same transformation. Functional images were smoothed with a Gaussian spatial filter of $5 \mathrm{~mm}$. The resulting images had cubic voxels of $4 \times 4 \times 4 \mathrm{~mm}^{3}$ and the final image resolution was $7.2 \times 7.2 \times 7.0$ $\mathrm{mm}^{3}$. Each task block was modeled by a single activation function derived by convolving the experimental paradigm with a Gaussian activation function taking into account the known delay of the haemodynamic response. Covariates of non-interest implemented a high-pass filter set at a period of $120 \mathrm{~s}$.

A standard significance threshold of $p<0.001$, cor-

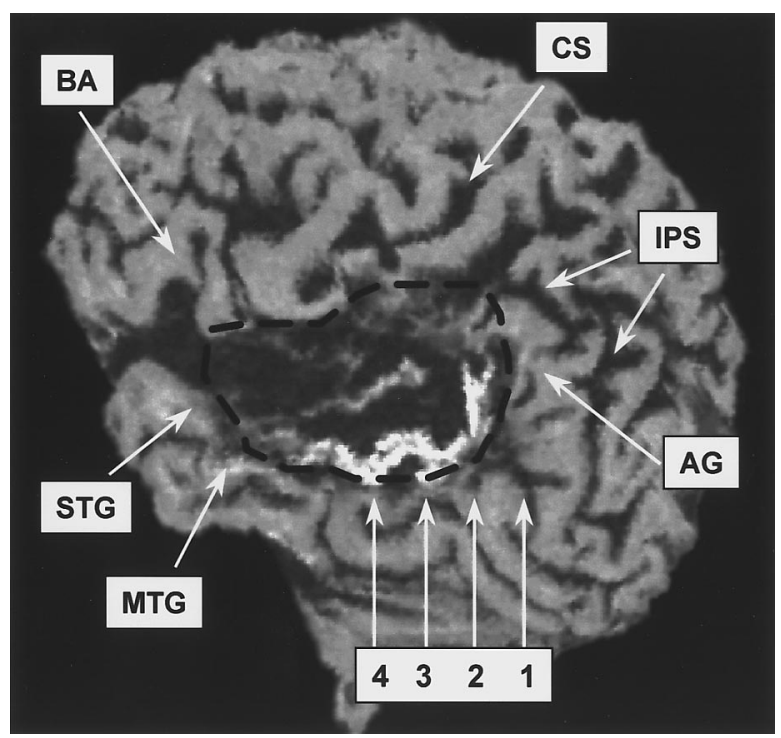

Fig. 1. Lateral view of the left hemisphere. The lesion affected part of the temporal and parietal perisylvian language cortex. The superior parietal lobule and the posterior part of the angular gyrus were spared. Numbers refer to the frontal sections in Fig. 2. BA: Broca's area; CS: central sulcus; IPS: intraparietal sulcus; AG: angular gyrus; STG: superior temporal gyrus; MTG: middle temporal gyrus. rected for multiple comparisons across the brain volume to $p<0.05$, was used in all tests. We first examined a global contrast for calculation relative to rest. Then we contrasted calculation with letter matching. Because each task was acquired in a distinct time series, this between-series contrast was framed as an interaction term: (calculation tasks - their resting periods)-(letter matching - its resting period). Finally, we determined significant differences between multiplication and subtraction using contrasts between those two operations (similarly framed as interaction terms). To focus only on activations, each contrast was masked by the appropriate contrast relative to rest, with masking threshold set at $p<0.05$ (e.g., the "multiplication $>$ subtraction" contrast was masked by "multiplication $>$ rest").

\subsection{Anatomical results}

As visible on a lateral view of the left hemisphere (Fig. 1), the lesion affected part of the classical perisylvian language cortex. The frontal lobe, including Broca's area, was spared. The superior temporal gyrus, part of the middle temporal gyrus, the lower part of the post-central gyrus, the supramarginal gyrus, and the anterior half of the angular gyrus were destroyed. The superior parietal lobule was intact. Frontal sections (Fig. 2) indicate that the cortex buried within the IPS, which makes the transition between the cortex of the superior and inferior parietal lobules, was partially spared. Within the IPS, the mesial cortical sheet was spared, as well as the posterior part of the lateral cortical sheet.

\subsection{Functional activation results}

\subsubsection{Behaviour}

Behavioural data collected during the scanning session and during the training session were collapsed. The patient made $2.5 \%(2 / 80)$ errors in the letter matching task (mean correct RT $=1564 \mathrm{~ms}$ ), $18.3 \%$ $(22 / 120)$ errors in multiplication verification (mean correct $\mathrm{RT}=1832 \mathrm{~ms})$, and $33.7 \%(27 / 80)$ errors in subtraction verification (mean correct RT $=1867 \mathrm{~ms}$ ). Her performance in multiplication verification was quite congruent with her score of close to $50 \%$ correct when writing down the results of simple multiplication problem, as described above: if she responded randomly on the $50 \%$ of trials in which she did not know the answer, one would expect an error rate of about $25 \%$ in multiplication verification, which is close to the observed error rate of $18.3 \%$. However, ATH's relatively poor performance in subtraction verification was unexpected, given her usually good performance in solving similar subtraction problems. We consider likely that this was largely due to the temporal con- 
straint of having to verify operations presented for a fixed duration of $2.5 \mathrm{~s}$ (her subtraction times in an unconstrained setting generally fell in the 5-10 s range).

\subsubsection{Calculation relative to rest}

When contrasting calculation and rest, a large network of areas was identified. The most intense activity was observed in the left and right intraparietal sulci and surrounding parietal cortex, the left and right dorsolateral prefrontal cortex, the anterior cingulate, and the cerebellum. Smaller foci were also observed in the occipital calcarine cortex, left and right inferior temporal gyri, and left and right inferior frontal gyri. Most importantly to our present purposes, the left posterior intraparietal cortex surrounding the lesion was clearly activated (Fig. 3), even if the extent and intensity of its activation was smaller than in homologous sectors of the right parietal lobe. At the level of the
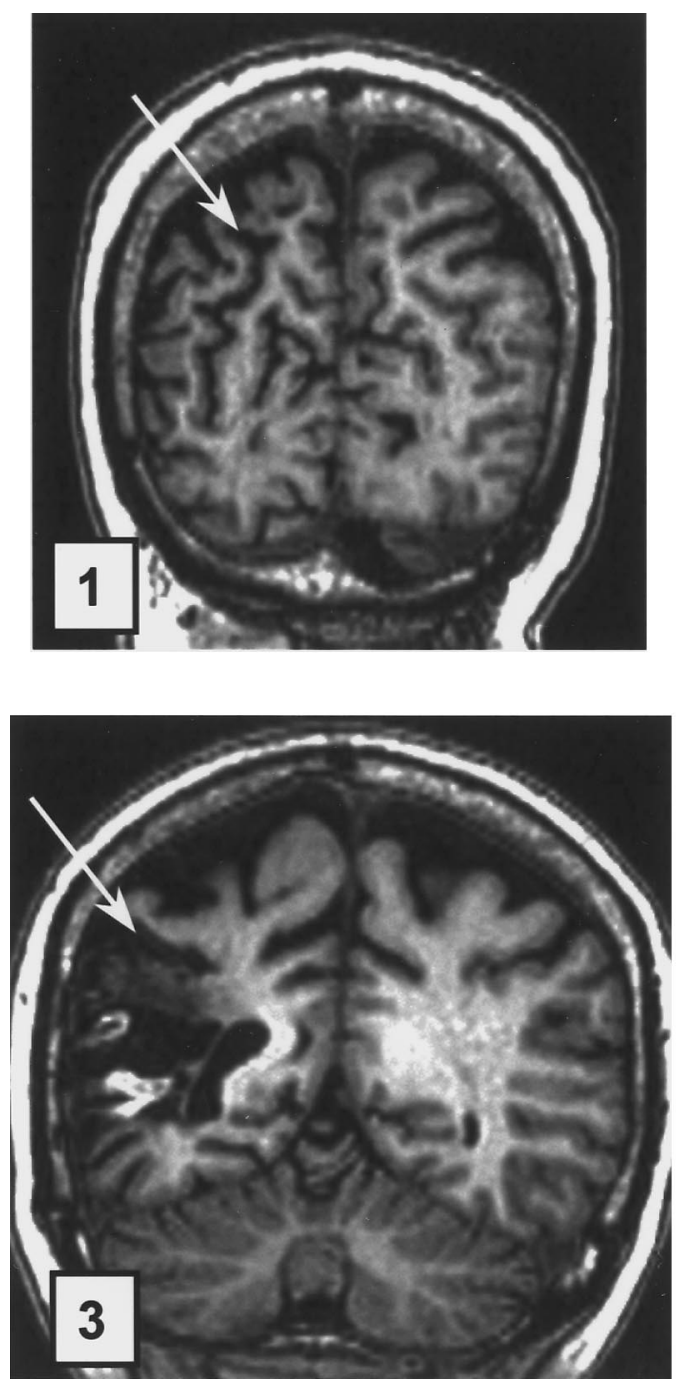

posterior part of the lesion (Fig. 3, top left), left-sided activity was restricted to the mesial intraparietal cortical sheet, while right-sided activity also extended to the lateral intraparietal cortex as well as to the lateral cortex of the IPL. More anteriorily (Fig. 3, top right), there was no significant left-sided activity, while there were still right-hemispheric activity in the lateral intraparietal and IPL cortex.

\subsubsection{Calculation relative to letter matching}

A relatively small subpart of this network showed significantly more activity during calculation than letter-matching: right intraparietal sulcus (Talairach coordinates $36,-52,40 ; Z=4.11$; Fig. 3, bottom left), right mesial occipital cortex (TC 4, -64, -12; $Z=5.32$ ), right precentral gyrus (TC 52, -4, 48; $Z=4.71$ ), and left dorsolateral prefrontal cortex (TC $-44,16,36, Z=4.98$; and TC $-44,28,28, Z=3.85$ ). Although left intraparietal activity was absent at this
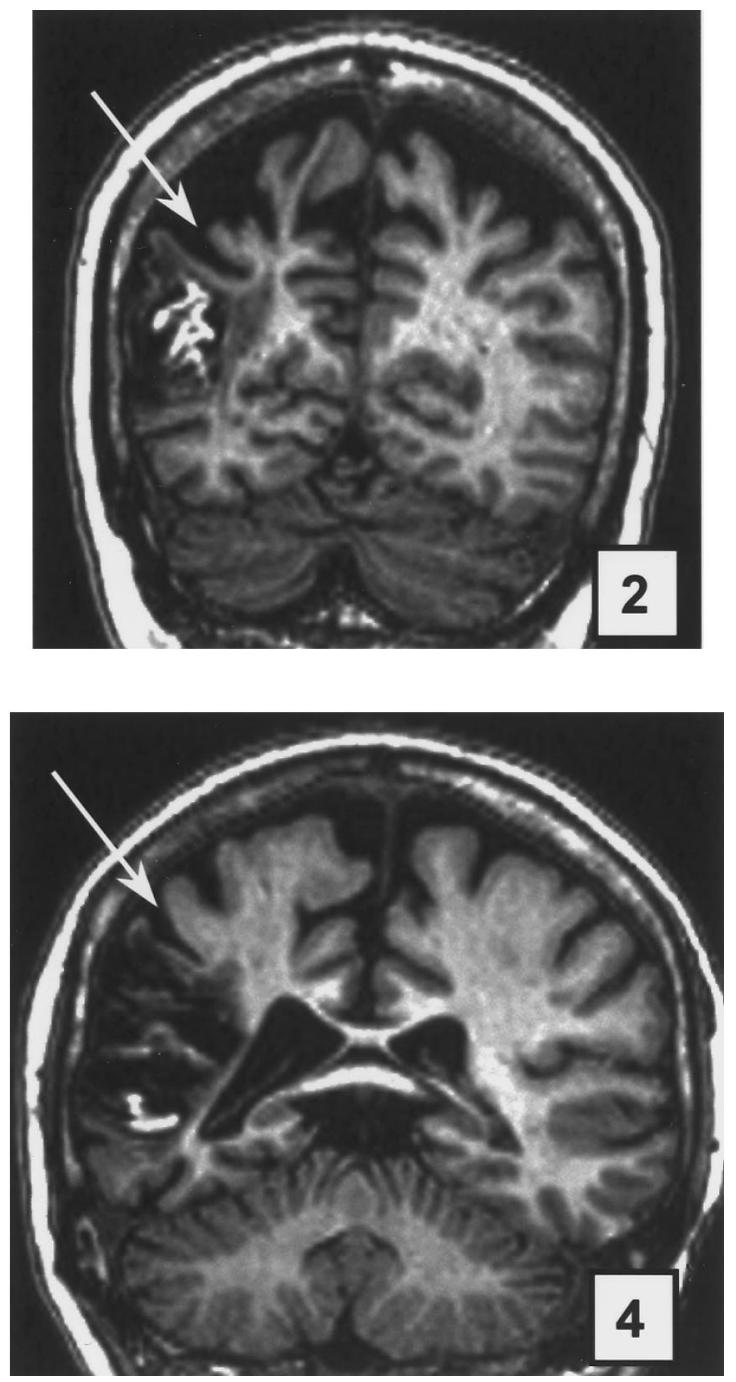

Fig. 2. Frontal sections through the posterior part of the lesion, as indicated in Fig. 1. Arrows point to the left intraparietal sulcus (IPS). The cortex buried within the IPS was partially spared, particularly its mesial cortical sheet. 
stringent level of significance, since this activation was predicted on an a priority basis, we also examined the images at a lower threshold (voxelwise $p<0.001$, cluster size $>2$ voxels). At this lower threshold, a small focus (6 voxels) was observed in the left posterior intraparietal cortex (TC $-32,-68,36 ; Z=4.64$; Fig. 3; bottom left).

\subsubsection{Multiplication versus subtraction}

A single region was more active during multiplication than during subtraction: the left posterior intraparietal sulcus, close to the parieto-occipital junction (TC $-12,-64,48 ; Z=4.27$ ). Conversely, a single region was more active during subtraction than during multiplication, in the right intraparietal sulcus (TC 28, $-48,56 ; Z=4.54)$.

\subsection{Discussion}

Anatomical images clearly confirmed the presence of a large left perisylvian lesion, with partial encroachment of the inferior parietal lobule. Functional images revealed that both left and right intraparietal cortices were functional and were significantly active during number processing. Some of these areas were shared with the letter matching task and may be involved in the shared spatial, attentional, or response requirements of the tasks. However, small bilateral intraparietal foci were found to be significantly more active during calculation than during the control task. This suggests that despite her lesion, the patient was recruiting a bilateral parietal circuit when verifying arithmetic problems. Indeed, the active areas clearly overlap with those observed in the intraparietal area in several previous brain-imaging studies of number processing $[7,21,22,34,35]$.

Interpretations of the comparisons between multiplication and subtraction should be formulated cautiously, because the patient's performance was relatively poor in both tasks. Still, the patient was far from responding randomly. We interpret her performance as indicating that she was struggling to solve the proposed operations on each trial, but that the fixed rate of presentation used often did not leave her enough time to complete them. If this interpretation is correct, the pattern of activation should still reflect the processes specifically involved in each task. Indeed, the functional imaging results do throw some light on the basis of the behavioural dissociation between multiplication and subtraction.

First, the subtraction task yielded more activity in the right intraparietal region than the multiplication task. Greater right intraparietal activation during subtraction than during multiplication was also observed in a recent fMRI study of normal subjects by Chochon et al. [7]. The coordinates of activation foci in these two studies fall within $1.5 \mathrm{~cm}$ of one another. In the study by Chochon et al., the right intraparietal region

\section{Calculation versus Rest}

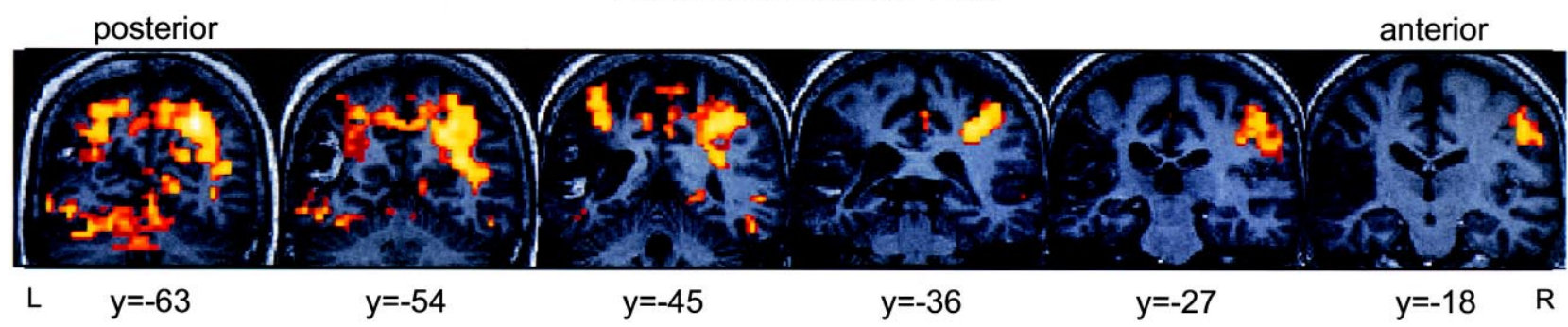

Calculation versus letter-matching

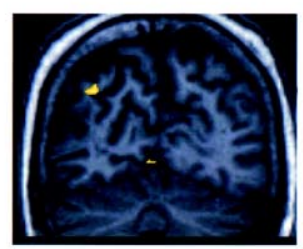

$y=-68$

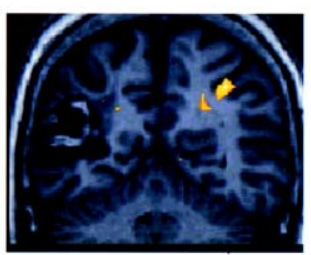

$y=-52$

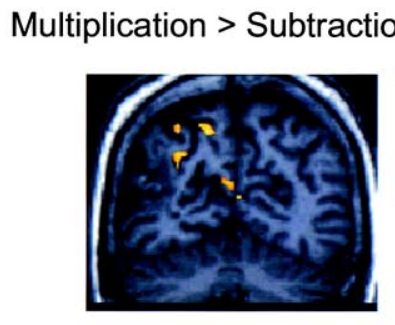

$y=-64$
Subtraction > Multiplication

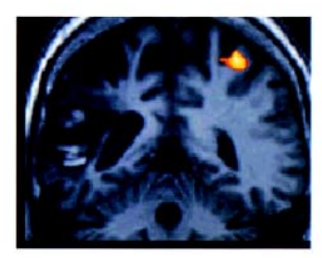

$y=-48$

Fig. 3. Functional magnetic resonance imaging in patient ATH during mental calculation. Bilateral intraparietal activations were observed when the two calculation tasks were contrasted to rest (top) and to letter matching (bottom left; for illustration purpose, those images were thresholded at $p<0.01)$. Left posterior intraparietal cortex showed greater activation during multiplication than during subtraction, while the converse contrast revealed a right intraparietal activation (bottom right; again, $p<0.01$ for illustration). 
was also the only region to show greater activity for number comparison than for number naming. We therefore suggest that this area can be associated with the internal manipulation of quantities (see also $[16,22])$. The fact that this region fell in the intact right hemisphere might explain why subtraction remained feasible by patient ATH, provided that she was given enough time.

Second, the multiplication task yielded more activity in the left posterior parietal area than the subtraction task, mesial to and above the angular gyrus. The coordinates of this activation fall very close to the peak reported by Chochon et al. [7] as showing greater activity during multiplication than during number comparison. Dehaene et al. [21] also reported greater activation in the vicinity of the angular gyrus during exact calculation, presumably relying of rote verbal memory, than during approximate calculation, presumably relying on quantity manipulation. One may suggest that the activation observed in patient ATH, which was contiguous to her lesion, might have represented the remains of a left-hemispheric network implicated in the verbal processing of numbers. Due to the extensive lesioning of left perisylvian language areas, this component might have become isolated and insufficient, in and of itself, for patient ATH to solve multiplication problems.

Finally, an account of all the activation differences between subtraction and multiplication purely in terms of a difference in effort requirements does not seem viable. If such was the case, one would only expect higher activation levels during subtraction as compared with multiplication. Actually, significant differences were observed in both directions. Particularly, the left posterior intraparietal sulcus, close to the parieto-occipital junction, was more active during multiplication than during subtraction. Furthermore, an interpretation based on effort and stress would probably predict an increased activation in prefrontal regions during subtraction, which was not observed.

\section{General discussion}

We reported the case of a patient, ATH, who suffered from aphasia, deep dyslexia, and acalculia, following a lesion in the left perisylvian area. Her deficit with spoken and written language extended to the domain of number processing. She showed a severe impairment in tasks involving numbers in a verbal format, such as reading aloud, writing to dictation, or responding verbally to questions of numerical knowledge. In contrast, her ability to manipulate non-verbal representations of numbers, i.e., Arabic numerals and quantities, was comparatively well preserved, as evidenced for instance in number comparison or number bisection tasks. This dissociated impairment of verbal and non-verbal numerical abilities entailed a differential impairment of the four arithmetic operations. ATH performed much better with subtraction and addition than with multiplication and division problems. We then attempted to correlate ATH's pattern of behaviour with the topography of her brain lesion, with a particular emphasis on the involvement of the parietal lobes. The lesion affected a subset of the classical language areas, including a substantial part of the left inferior parietal lobule. However, the posterior part of the left angular gyrus and some of the underlying intraparietal cortex were anatomically intact. Functional MRI during subtraction and multiplication verification tasks indicated that the intact left angular and intraparietal cortex was significantly activated during problem verification, although less strongly than the analogous right-hemispheric areas. When comparing multiplication and subtraction, we observed that subtraction evoked a right parietal network, while multiplication activated the vicinity of the left angular gyrus.

The existence of double behavioural dissociations between elementary arithmetic abilities depending on their reliance on verbal processes suggests that partly distinct cerebral circuits are in charge of the verbal and quantitative aspects of number processing, which will be discussed in turn.

Regarding the verbal component of arithmetic processing, the triple code model postulates a circuit that includes both cortical areas and cortico-subcortical loops involved in the retrieval of verbal automatisms. In patient BOO [19], the arithmetic impairment presumably resulted from a subcortical lesion responsible for a selective deficit of automatic speech extending beyond the numerical domain. In contrast, patient ATH's lesion affected the cortical language areas. Thus, patients BOO and ATH may represent, respectively, subcortical and cortical variants of what may be called "verbal anarithmetia". Accordingly, they both displayed the same general pattern of dissociation, namely, impaired multiplication with relatively spared subtraction. Such sparing of subtraction as compared with presumably more "verbal" operations is not an isolated finding, and has been previously reported in other patients $[14,27,33]$. A similar pattern of dissociation has also been observed in patients with still another type of brain lesion. Some patients with pure alexia $[9,10,32]$, while they could solve normally problems that were presented in the auditory modality, were impaired with visually presented problems. However, this impairment affected multiplication much more severely than subtraction. We suggested that this discrepancy between operations reflects the fact that these pure alexic patients, due to their left inferior temporal lesion, were unable to translate Arabic operands 
into words. Hence they were unable to access multiplication facts stored as verbal associations. In contrast, subtraction problems could be solved by directly accessing and manipulating the quantities represented by Arabic operands, without requiring a prior encoding in verbal format. In brief, a disproportionate impairment of multiplication as compared with subtraction may be taken as an index of the verbal origin of anarithmetia, and can result from different functional mechanisms, corresponding to distinct lesion sites: (1) in pure alexia following left infero-temporal lesions, Arabic stimuli cannot be translated into words, a prerequisite for retrieving stored multiplication facts; (2) lesions affecting the language cortex, as in patient ATH, can impede the ability to represent multiplication problems as word strings; (3) left subcortical lesions can interfere with the retrieval of stored verbal automatisms, including familiar multiplication facts.

According to the triple-code model, the opposite dissociation between operation types, i.e., preserved multiplication with severely impaired subtraction, results from deficits affecting the semantic or quantitative component of elementary arithmetic processing. In such cases, anarithmetia is part of a more general impairment of quantity manipulation, visible for instance in number comparison or bisection tasks [19]. It is essential to note that this type of "semantic anarithmetia" can occur in the absence of any impairment of reading, writing, or more generally of any deficit in the verbal domain. It is often associated with other features of Gerstmann's syndrome, and results from lesions classically affecting the inferior parietal lobule, possibly the cortex surrounding the IPS [20,23,38]. Patient ATH did not present this semantic type of anarithmetia, and indeed showed none of the other deficits that, in addition to acalculia, constitute Gerstmann's syndrome: she had no left-right confusion and no finger agnosia, and her agraphia was of the aphasic type, while the agraphia associated with Gerstmann's syndrome is generally of the apraxic type, due to the more dorsal site of parietal lesions [2,28]. Thus, the study of ATH's lesion may provide useful "negative evidence" for delineating the critical site of lesions responsible for semantic anarithmetia.

On the basis of anatomical and functional evidence, we showed that in patient ATH's left hemisphere, the posterior angular and intraparietal cortex was anatomically spared, but also functionally activated during arithmetic tasks (although this activation was much weaker than in the right hemisphere). Since, contrary to some other patients with dominant parietal lesions, ATH did not show the Gerstmann type of acalculia, but rather an anarithmetia contingent on her verbal deficit, one may speculate that this patch of spared left parietal cortex corresponds at least in part to the parietal structures devoted to quantity processing. Still, the clearest conclusion that can be drawn from the anatomical and fMRI study is that ATH's intact right parietal lobe probably played a important role in her preserved numerical abilities. This region indeed showed strong activations during arithmetic tasks, including activations specific to such tasks. It was also the only region that was found to be more active during subtraction than during multiplication. This pattern of right parietal activation is similar to that reported in normal subjects. While it is often difficult to establish whether structures activated in normal subjects are actually necessary to the performance of the experimental task, the fact that patient ATH suffered from a large left-sided lesion, with very reduced adjacent activations, suggests that the right parietal lobe played an important role in her preserved arithmetic abilities, particularly quantity-based procedures such as subtraction or number comparison.

\section{Acknowledgements}

We thank patient ATH for her participation, and Ms. D. Sosson for her help in the language assessment.

\section{References}

[1] Alexander GE, Delong MR, Strick PL. Parallel organization of functionally segregated circuits linking basal ganglia and cortex. Annual Review of Neuroscience 1986;9:357-81.

[2] Alexander MP, Fischer RS, Friedman R. Lesion localization in apractic agraphia. Archives of Neurology 1992;49:246-51.

[3] Alexander MP, Naeser MA, Palumbo CL. Correlations of subcortical CT lesion sites and aphasia profiles. Brain 1987;110:961-91.

[4] Binder JR, Mohr JP. The topography of callosal reading pathways. A case-control analysis. Brain 1992;115:1807-26.

[5] Boysen ST, Capaldi EJ, editors. The Development of Numerical Competence: Animal and Human Models. Hillsdale, NJ: Erlbaum, 1993.

[6] Buckley PB, Gillman CB. Comparison of digits and dot patterns. Journal of Experimental Psychology 1974;103:1131-6.

[7] Chochon F, Cohen L, van de Moortele PF, Dehaene S. Cortical and subcortical circuits for mental arithmetic: functional neuroimaging of bilateral intraparietal, frontal, cingular and caudate contributions to various calculation tasks. Journal of Cognitive Neuroscience 1999;11:617-30.

[8] Church RM, Meck WH. The numerical attribute of stimuli. In: Roitblat HL, Bever TG, Terrace HS, editors. Animal Cognition. Hillsdale, NJ: Erlbaum, 1984.

[9] Cohen L, Dehaene S. Number processing in pure alexia: the effect of hemispheric asymmetries and task demands. NeuroCase 1995;1:121-37.

[10] Cohen L, Dehaene S. Calculating without reading: unsuspected residual abilities in pure alexia. Cognitive Neuropsychology, in press.

[11] Cohen L, Dehaene S, Verstichel P. Number words and number non-words: a case of deep dyslexia extending to arabic numerals. Brain 1994;117:267-79. 
[12] Cohen L, Verstichel P, Dehaene S. Neologistic jargon sparing numbers: a category specific phonological impairment. Cognitive Neuropsychology 1997;14:1029-61.

[13] Coltheart M, Patterson KE, Marshall JC. Deep dyslexia, London, 1980.

[14] Dagenbach D, McCloskey M. The organization of arithmetic facts in memory: evidence from a brain-damaged patient. Brain and Cognition 1992;20:345-66.

[15] Damasio AR, Damasio H. Anatomical basis of pure alexia. Neurology 1983;33:1573-83.

[16] Dehaene S. The organization of brain activations in number comparison: event-related potentials and the additive-factors methods. Journal of Cognitive Neuroscience 1996;8:47-68.

[17] Dehaene S, Changeux JP. Development of elementary numerical abilities: a neuronal model. Journal of Cognitive Neuroscience 1993;5:390-407.

[18] Dehaene S, Cohen L. Towards an anatomical and functional model of number processing. Mathematical Cognition 1995; 1:83-120.

[19] Dehaene S, Cohen L. Cerebral pathways for calculation: double dissociations between Gerstmann's acalculia and subcortical acalculia. Cortex 1997;33:219-50.

[20] Dehaene S, Dehaene-Lambertz G, Cohen L. Abstract representations of numbers in the animal and human brain. Trends in Neuroscience 1998;21:355-61.

[21] Dehaene S, Spelke E, Pinel P, Stanescu R, Tsivkin S. Sources of mathematical thinking: behavioral and brain-imaging evidence. Science 1999;284:970-4.

[22] Dehaene S, Tzourio N, Frak V, Raynaud L, Cohen L, Mehler J, Mazoyer B. Cerebral activations during number multiplication and comparison: a PET study. Neuropsychologia 1996;34:1097-106.

[23] Delazer M, Benke T. Arithmetic facts without meaning. Cortex 1997;33:697-710.

[24] Grafman J, Passafiume D, Faglioni P, Boller F. Calculation disturbances in adults with focal hemispheric damage. Cortex 1982;18:37-49.

[25] H'caen H, Angelergues R, Houillier S. Les variétés cliniques des acalculies au cours des lésions rétro-rolandiques: approche statistique du problème. Revue Neurologique 1961;105:85-103.

[26] Henschen SE. Klinische und Anatomische Beitraege zur Pathologie des Gehirns. Stockholm: Nordiska Bokhandeln, 1920.

[27] Lampl Y, Eshel Y, Gilad R, Sarova-Pinhas I. Selective acalculia with sparing of the subtraction process in a patient with left parietotemporal hemorrhage. Neurology 1994;44:1759-61.

[28] Lang C. Die agraphie des gerstmann-syndroms: versuch einer
Charakterisierung. Fortschritte in der neurologie und psychiatrie 1994;62:155-63.

[29] Lecours AR, Lhermitte F, Bryans B. Aphasiology. London: Baillière Tindall, 1983.

[30] McCloskey M. Cognitive mechanisms in numerical processing: evidence from acquired dyscalculia. Cognition 1992;44:107-57.

[31] McCloskey M, Macaruso P, Whetstone T. The functional architecture of numerical processing mechanisms: defending the modular model. In: The Nature and Origins of Mathematical Skills. Amsterdam: J.I.D. Campbell. North Holland, 1992. p. 493-537.

[32] McNeil JE, Warrington EK. A dissociation between addition and subtraction within written calculation. Neuropsychologia 1994;32:717-28.

[33] Pesenti M, Seron X, Van Der Linden, M. Selective impairment as evidence for mental organisation of arithmetical facts: BB, a case of preserved subtraction? Cortex 1994;30:661-71.

[34] Roland PE, Friberg L. Localization of cortical areas activated by thinking. Journal of Neurophysiology 1985;53:1219-43.

[35] Rueckert L, Lange N, Partiot A, Appollonio I, Litvar I, Le Bihan D, Grafman J. Visualizing cortical activation during mental calculation with functional MRI. NeuroImage 1996;3:97103.

[36] Shallice T. From Neuropsychology to Mental Structure. Cambridge: Cambridge University Press, 1988.

[37] Starkey P, Spelke ES, Gelman R. Detection of intermodal numerical correspondences by human infants. Science 1983;222:179-81.

[38] Takayama Y, Sugishita M, Akiguchi I, Kimura J. Isolated acalculia due to left parietal lesion. Archives of Neurology 1994;51:286-91.

[39] Warrington EK. The fractionation of arithmetical skills: A single case study. Quarterly Journal of Experimental Psychology 1982;34A:31-51.

[40] Whalen J, Gallistel CR, Gelman R. Non-verbal counting in humans: the psychophysics of number representation. Psychological Science 1999;10:130-7.

[41] Wynn K. Addition and subtraction by human infants. Nature 1992;358:749-50.

\section{Further reading}

Link SW. Modelling imageless thought: the relative judgment theory of numerical comparisons. Journal of Mathematical Psychology 1990;34:2-41. 